\title{
ГЕОЭКОЛОГИЯ
}

\section{Влияние расхода воздуха сверх нормы на потребляемую и полезную мощность источника ТЯГИ}

\author{
А.И. Петров \\ ГИ УрО РАН, 614007, Пермь, ул. Сибирская, 78а \\ ООО «ЗУМК-Инжиниринг», 614000, Пермь, ул. 25-го Октября, 2 \\ E-mail: alex231287@yandex.ru \\ (Статья поступила в редакцию 14 марта 2013 г.)
}

\begin{abstract}
Представлены результаты исследования экономичного функционирования системы вентиляции подземного горного предприятия. Его целью явилась необходимость сокращения финансовых затрат на ее функционирование за счет снижения потребления электроэнергии вентиляторными установками. В статье рассматривается главный фактор, служащий причиной высокого уровня энергопотребления,- расход воздуха сверх нормы.

Ключевые слова: рудник, шахта, проветривание, вентиляция, энергопотребление, электроэнергия, система вентилячии, расход воздуха, вентиляторная установка.
\end{abstract}

Объем потребляемой электроэнергии с целью эффективного проветривания рудника составляет существенную, а иногда большую, часть энергопотребления подземного горного предприятия $[3,4,8]$. Он оказывает значительное влияние на издержки производства; увеличение себестоимости добываемого полезного ископаемого, рост количества участков месторождения, попадающих в разряд забалансовых, и в итоге - на сокращение прибыли горного предприятия. Анализ публикаций показал, что в настоящее время пути и способы нормализации проветривания подземных рудников изучены недостаточно, а рациональные и экономичные методы и средства их проветривания не разработаны [1].

Повышенный уровень энергопотребления системой вентиляции горного предприятия во многом определяется перерасходом электроэнергии сверх нормы, требуемой для обеспечения необходимым количеством воздуха всех потребителей. В соответствии с видами и объемом горных работ, проводимых в подземных горных выработках, в отдельные рабочие зоны и рудник в целом требуется подавать определенное количество воздуха в единицу времени. Для чего источник тяги (ГВУ, ВВУ, ПВВУ, ППВУ, ВМП и т.д.) должен быть способен создать необходимую разность давлений.

Потребляемая источником тяги мощность $N$ (кВт) будет определяться по формуле [2]

$$
N=\frac{Q_{\mathrm{UT}} \cdot h_{\mathrm{UT}}}{1000 \cdot \eta_{\mathrm{BV}} \cdot \eta_{\mathrm{\Pi}}}
$$

где $Q_{\text {ит }}$ - производительность источника тяги, ${ }^{3} / \mathrm{c} ; h_{\text {ит }}-$ развиваемое источником тяги давление, Па; $\eta_{\mathbb{B y}}$ - коэффициент полезного действия вентиляторной установки в указанном режиме работы, отн.ед.; $\eta_{\Pi}-$ коэффициент полезного действия привода вентилятор- 
ной установки в указанном режиме работы, отн.ед.

При этом действительная полезная мощность $N_{\text {д }}$ (кВТ), развиваемая источником тяги, определяется по формуле [6]

$$
N_{\mathrm{A}}=\frac{Q_{\mathrm{MT}} \cdot h_{\mathrm{UT}}}{1000},
$$

где $Q_{\text {ит }}$ - подача (производительность) источника тяги, $\mathrm{M}^{3} / \mathrm{c} ; h_{\mathrm{MT}}-$ развиваемое источником тяги давление, Па.

Кривая характеристики любой вентиляционной сети описывается уравнением $[9,6,7]$

$$
h=R \cdot Q^{2} \text {, }
$$

где $h$ - перепад давления вентиляционной сети, Па; $R$ - общее аэродинамичекое сопротивление вентиляционной сети, $\mathrm{H} \cdot \mathrm{c}^{2} / \mathrm{M}^{8} ; Q-$ общий расход воздуха в вентиляционной сети, $\mathrm{m}^{3} / \mathrm{c}$.

Отсюда развиваемое источником тяги давление $h_{\mathrm{UT}}$, необходимое для того, чтобы обеспечить рассматриваемую вентиляционную сеть требуемым уровнем расхода воздуха, пропорциональным квадрату величины производительности источника тяги $Q_{\mathrm{MT}}$, а также общему аэродинамическому сопротивлению вентиляционной сети $R$ :

$$
h_{\mathrm{WT}}=R \cdot Q_{\mathrm{WT}}{ }^{2} \text {. }
$$

Полезная мощность $N_{\text {д }}$, развиваемая источником тяги:

$$
N_{\mathrm{A}}=\frac{R \cdot Q_{\mathrm{UT}}{ }^{3}}{1000} \text {. }
$$

Анализ формулы показывает, что величина действительной полезной мощности ис-

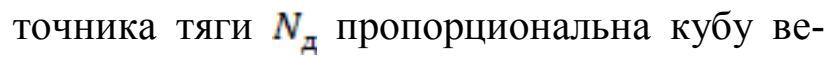
личины производительности данного источника тяги $Q_{\text {ит: }}$ :

$$
N_{\mathrm{A}} \sim Q_{\mathrm{MT}}{ }^{3} \text {. }
$$

Запись формулы именно в таком виде более приемлема в данном случае, чем традиционная запись данного выражения в виде произведения величины давления, развиваемого источником тяги, и величины его производительности, деленной на тысячу. Это объясняется тем, что на данном этапе нет необходимости в поиске рабочей точки источника тяги, а значит, и нет необходимости в вычислении развиваемого источником тяги давления. С другой стороны, данное выражение наглядно отражает первичные условия поставленной задачи: аэродинамическое со- противление вентиляционной сети и расход воздуха, который должен быть обеспечен в данной вентиляционной сети.

Представим абсолютную величину перерасхода воздуха в какой-либо вентиляционной сети следующим образом:

$$
\Delta Q=Q_{\mathrm{UT}}-Q_{\mathrm{Tp}},
$$

где $Q_{\text {тр }}$ - количество воздуха, требуемое для обеспечения эффективного проветривания всех фактических на рассматриваемый момент потребителей воздуха.

$$
\begin{gathered}
\text { Тогда } \\
N_{\mathrm{p}}=\frac{R \cdot\left(Q_{\mathrm{Tp}}+\Delta Q\right)^{3}}{1000}= \\
=\frac{R \cdot\left(Q_{\mathrm{Tp}}{ }^{3}+3 \cdot Q_{\mathrm{Tp}}{ }^{2} \cdot \Delta Q+3 \cdot Q_{\mathrm{Tp}} \cdot \Delta Q^{2}+\Delta Q^{3}\right)}{1000} .
\end{gathered}
$$

Абсолютный перерасход полезной мощности:

$$
\Delta N_{\mathrm{A}}=N_{\mathrm{A}}-N_{\mathrm{Tp}},
$$

где $N_{\text {тр }}$ - действительная полезная мощность, необходимая для подачи в вентиляционную сеть количества воздуха, требуемого для обеспечения эффективного проветривания всех фактических на рассматриваемый момент потребителей.

$$
N_{\text {тр }}=\frac{R \cdot Q_{\text {тр }}{ }^{3}}{1000} .
$$

Относительный перерасход полезной мощности:

$$
\Delta N_{\% 6}=\frac{\left(N_{\mathrm{A}}-N_{\mathrm{Tp}}\right) \cdot 100}{N_{\text {тр }}} .
$$

Относительный и абсолютный перерасход воздуха:

$$
\Delta Q_{96}=\frac{\Delta Q \cdot 100}{Q_{\text {тp }}}, \quad \Delta Q=\frac{\Delta Q_{96} \cdot Q_{\text {тp }}}{100}
$$

Выразим относительный перерасход полезной мощности через относительный перерасход воздуха:

$$
\begin{aligned}
& \Delta N_{\mathrm{q}}=\frac{\left(N_{\mathrm{A}}-N_{\mathrm{Tp}}\right) \cdot 100}{N_{\mathrm{Tp}}}= \\
& =3 \cdot \Delta Q_{96}+\frac{3 \cdot \Delta Q_{q 6}{ }^{2}}{100}+\frac{\Delta Q_{96}{ }^{3}}{10000} .
\end{aligned}
$$

Анализ полученной математической зависимости относительного перерасхода полез-

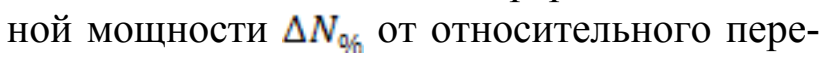
расхода воздуха $\Delta Q_{g, h}$ показал: 


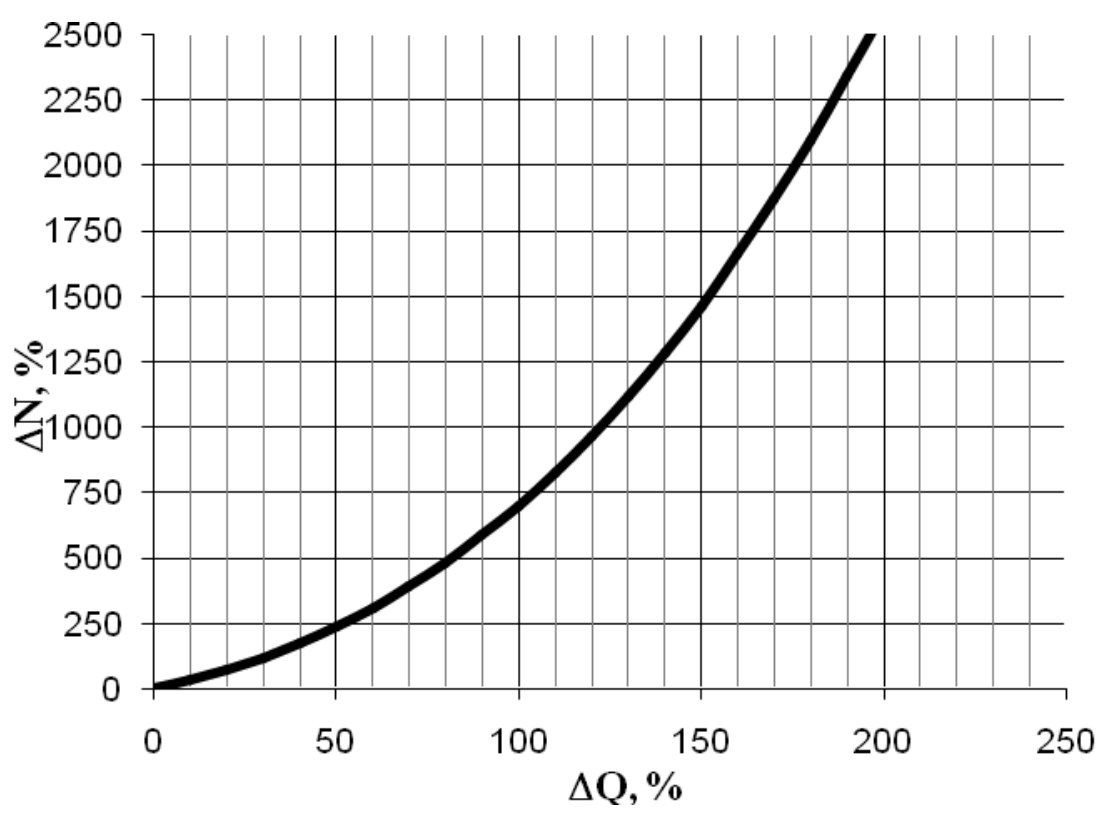

График зависимости величины относительного перерасхода полезной мощности $\triangle N_{\text {乌̆ }}$ ГВУ от относительного перерасхода воздуха $\Delta Q_{q}$

- величина относительного перерасхода полезной мощности не зависит от характеристики вентиляционной сети рудника, т. е. от ее аэродинамического сопротивления;

- величина относительного перерасхода полезной мощности не зависит от характеристики источника тяги;

- величина относительного перерасхода полезной мощности определяется только величиной относительного перерасхода воздуха.

Зависимость относительного перерасхода полезной мощности $\Delta N_{\text {क़h }}(\%)$ от относительного перерасхода воздуха $\Delta Q_{0, h}(\%)$ будет иметь вид, изображенный на графике.

Абсолютный перерасход полезной мощности, выраженный через абсолютный перерасход воздуха, можно записать следующим образом:

$$
\begin{aligned}
& \Delta N_{\mathrm{A}}=N_{\mathrm{A}}-N_{\mathrm{Tp}}= \\
= & \frac{R}{1000}\left(3 \cdot Q_{\mathrm{Tp}}^{2} \cdot \Delta Q+3 \cdot Q_{\mathrm{Tp}} \cdot \Delta Q^{2}+\Delta Q^{3}\right) .
\end{aligned}
$$

В отличие от относительного, абсолютный перерасход полезной мощности будет являться функцией от аэродинамического сопротивления $R$ вентиляционной сети и количества воздуха $Q_{\text {тр }}$, требуемого для обеспечения эффективного проветривания всех фактических на рассматриваемый момент потребителей воздуха в рассматриваемой вентиляционной сети.
Полученная зависимость относительного перерасхода полезной мощности $\Delta N_{\text {g, }}$ от относительного перерасхода воздуха $\Delta Q_{9, h}$ была проверена на математической модели вентиляционной сети южного участка рудника объекта «Горнодобывающее и перерабатывающее предприятие на месторождении "Нежданинское"», построенной с помощью программного комплекса «АэроСеть 2D», позволяющего выполнять расчет распределения потоков воздуха по вентиляционной сети.

Для построенной модели вентиляционной сети рудника в соответствии с нормативными расходами воздуха во всех предполагаемых рабочих зонах был подобран источник тяги, вентиляторная установка ВОД-18. Путем изменения скорости вращения рабочего колеса вентиляторной установки от 500 до 1000 об/мин с интервалом изменения 50 об/мин, были получены данные, отражающие зависимость изменения величины подачи вентиляторной установки $Q$ и ее активной мощности $N_{\text {a }}$ от скорости вращения рабочего колеса $n$ (таблица). Для рассматриваемых режимов работы вентиляторной установки были рассчитаны величины абсолютного $\Delta Q$ и относительного $\Delta Q_{g, ~}$ перерасходов воздуха, а также абсолютного $\Delta N$ и относительного $\Delta N_{\text {o, }}$ перерасхода мощности. 
Полученная таким путем зависимость «относительный перерасход полезной мощности $\Delta N_{\text {ф̆ }}$ источника тяги - относительный перерасход воздуха $\Delta Q_{\text {gh }} \gg$ идентична ранее полученной. Графические изображения указанных зависимостей будут полностью совпадать, тем самым подтверждая друг друга.

\begin{tabular}{|c|c|c|c|c|c|c|}
\hline$n$, об/мин & $Q, \mathrm{~m}^{3} / \mathrm{мин}$ & $N_{\mathrm{a}}, \mathrm{\kappa BT}$ & $\Delta Q, \mathrm{M}^{3} / \mathrm{Mин}$ & $\Delta Q_{g h}, \%$ & $\Delta N$, кВ Т & $\Delta N_{g, h}, \%$ \\
\hline 500 & 5124 & 9,3 & 0 & 0 & 0 & 0 \\
\hline 550 & 5637 & 12,4 & 513 & 10,01 & 3,1 & 33,33 \\
\hline 600 & 6149 & 16,1 & 1025 & 20,00 & 6,8 & 73,12 \\
\hline 650 & 6662 & 20,4 & 1538 & 30,01 & 11,1 & 119,35 \\
\hline 700 & 7174 & 25,5 & 2050 & 40,00 & 16,2 & 174,19 \\
\hline 750 & 7687 & 31,4 & 2563 & 50,01 & 22,1 & 237,63 \\
\hline 800 & 8199 & 38,1 & 3075 & 60,01 & 28,8 & 309,68 \\
\hline 850 & 8711 & 45,7 & 3587 & 70,00 & 36,4 & 391,39 \\
\hline 900 & 9224 & 54,2 & 4100 & 80,01 & 44,9 & 482,79 \\
\hline 950 & 9736 & 63,8 & 4612 & 90,00 & 54,5 & 586,02 \\
\hline 1000 & 10249 & 74,4 & 5125 & 100,01 & 65,1 & 700,00 \\
\hline
\end{tabular}

Таким образом, величина действительной полезной мощности, развиваемой источником тяги (вентиляторной установкой), главным образом определяется величиной подачи воздуха в вентиляционную сеть, на которую работает данный источник тяги. Величина прироста полезной мощности не пропорциональна соответствующему ей приросту расхода воздуха, и даже небольшое увеличение производительности источника тяги сверх нормы приводит к существенному перерасходу полезной и потребляемой мощности.

\section{Библиографический список}

1. Алыменко Н.И., Минин В.В. Вентиляторные установки и их применение / УрО РАН. Екатеринбург, 1999, 224 с.

2. Алыменко Н.И. Исследование работы вентиляторных установок главного проветривания и разработка мероприятий по повышению эффективности их эксплуатации примени- тельно к условиям калийных рудников. Дис. ... канд. техн. наук. Л., 1982.

3. Ковалевская В.И., Бочаров Е.П., Емельянов $B . A$. Анализ работы действующих вентиляторных установок шахт // Безопасность труда в промышленности. 1969. №11. С. 17-18.

4. Луговский С.И. и др. Совершенствование разработки и вентиляции рудников. М.: Недра, 1968.303 с.

5. Мохирев Н.Н., Радько В.В. Инженерные расчеты вентиляции шахт. Строительство. Реконструкция. Эксплуатация. М.: ООО «Недра-Бизнесцентр», 2007. 324 с.

6. Мохирев Н.Н., Попов А.С. Расчеты при проектировании вентиляции шахт: учеб.-метод. пособие. Пермь: Изд-во Перм. гос. техн. унта, 2006. $317 \mathrm{c}$.

7. Скочинский А.А., Комаров В.Б. Рудничная вентиляция. М.: Углетехиздат, 1959. $632 \mathrm{c.}$

8. Шиак Г.В. и др. Состояние проветривания североуральских рудников // Вопросы горной электромеханики. М., 1969. Вып. 1. С. 74-83.

9. Ушаков К.3., Бурчаков А.С., Пучков Л.А., Медведев И.И. Аэрология горных предприятий. - М.: Недра, 1987. 421с. 


\title{
Effect of excessive air consumption on power consumption of fan
}

\author{
A.I. Petrov \\ Mining institute of the Ural branch of the Russian Academy of Scinces, \\ 614007, Perm, Sibirskaya St., 78a \\ LLC «ZUMK-Engineering», 614000, Perm, 25 October St., 2 \\ E-mail: alex231287@yandex.ru
}

The article deals with the results of the research on the cost-effective functioning of the ventilation system of underground mine. This work was focused to development of a method of reducing the financial cost of the exploitation of the ventilation systems of the mining enterprise by the reduction of electric power consumption of the fan units. The aim of study was to identify the main factors forming the values of energy consumption of fan system. The flow rate above the normal air consumption was considered as the main factor, which causes the high level of power consumption of ventilation system of mining enterprise.

Key words: mine, ventilation, energy consumption, ventilation system, air flow, fan.

\section{References}

1. Alymenko N.I., Minin V.V., 1999. Ventilyatornye ustanovki i ikh primenenie [Fan systems and teir usage]. Yekaterinburg, UB RAS, p. 224.

2. Alymenko N.I., 1982. Issledovanie raboty ventilyatornykh ustanovok glavnogo provetrivaniya I razrabotka meropriyatiy po povysheniyu effektivnosti ikh ekspluatatsii primenitelno $\mathrm{k}$ usloviyam kaliynykh rudnikov [Study of functioning of fan system of main ventilation and design the measures for their effective exploitation according to the conditions of potash mines]. Diss, na sosisk. uch. st. kandidata tekh. nauk, Leningrad.

3. Kovalevskaya V.I., Bocharov E.P., and Emelyanov V.A., 1969. Analys raboty deystvuyushchikh ventilyatsionnykh ustanovok shakht [Analysis of functioning of the operating ventilation systems of mines]. Bezopasnost truda $\mathrm{v}$ promyshlennosti. 11:17-18.

4. Lugovskiy S.I. et al., 1968. Sovershenstvovanie razrabotki i ventilyatsii rudnikov [Improvement of design and ventilation of mines]. Moscow, Nedra, p. 303.

5. Mokhirev N.N., Radko V.V., 2007. Inzhenernye raschoty ventilyatsii shakht. Stroitelstvo. Rekonstruktsiya. Ekspluatatsiya. [Engineering computation of mine ventilation. Construction. Reconstruction. Exploitation.]. Moscow, NedraBisnestsentr, p. 324.

6. Rashchety pri proektirovanii ventilyatsii shakht [Computation for mine ventilation design]. Mokhirev N.N., Popov A.S., 2006. Perm, Perm State Techn. Univ. Publ., p. 317.

7. Skochinskiy A.A., Komarov V.B., 1959. Rudnichnaya ventilyatsiya [Mine ventilation]. Moscow, Ugletekhizdat, p. 632.

8. Shpak G.V. et al., 1969. Sostoyanie provetrivaniya Severouralskikh rudnikov [Condotion of ventilation of the North Ural's mines]. Voprosy gornoy elektromekhaniki. 1:74-83.

9. Ushakov K.Z., Burchakov A.S., Puchkov L.A., and Medvedev I.I., 1987. Aerologiya gornykh predpriyatiy [Aerology of mining enterprise]. Moscow, Nedra, p. 421. 